\title{
A MODEL FOR STELLAR CONVECTION AND SPECTRAL LINE ASYMMETRIES
}

\author{
H. M. Antia \\ Tata Institute of Fundamental Research \\ Homi Bhabha Road, Bombay 400 005, INDIA
}

\begin{abstract}
A model for stellar convection zones based on linear convective modes using a nonlocal mixing length theory is developed to study the spectral line asymmetries and the line shifts resulting from convective motions in the stellar photospheric region. The amplitudes of these linear convective modes is estimated by requiring the convective flux due to a linear superposition of such modes to reproduce the convective flux in the mixing length model. To study the spectral line asymmetries the convective mode with the largest amplitude in the photospheric line formation region is chosen to represent the stellar velocity field and the accompanying intensity fluctuations. Synthetic spectral line profiles are obtained by summing locally symmetric profiles over the stellar disk according to the local Doppler velocity and intensity fluctuations. The resulting line bisector shapes and the line shifts are compared with observations for $\alpha$-Cen B. It is found that while the simple model proposed here can explain either the line shifts or the line bisector shape reasonably well, it fails to explain both these characteristics simultaneously.
\end{abstract}

\section{Introduction}

The asymmetry and the accompanying line shift in the solar photospheric lines has been known for quite some time. These shifts and asymmetries have been ascribed to the correlated velocity and brightness patterns due to solar convection. Several studies have been made to detect asymmetries in other stars and some results have been obtained (Gray and Toner 1985, Dravins 1987). The asymmetry can be most conveniently characterized in terms of the line bisector which is the loci of points midway between equal intensity points on either side of the line.

A number of attempts have been made to model the convective motions in stars to reproduce the observed asymmetries. These models can be classified into two categories. Firstly there are simple two to four-stream models where the stellar surface is divided into two to four components of different characteristic brightness and velocity. The synthetic line profile is obtained as a summation of line profiles from these components appropriately weighted for their area coverage. By choosing the appropriate combinations of velocity and intensity over each of these components it is possible to model a wide variety of line bisector shapes. Such models are essentially ad hoc and the parameters are chosen to get the "best" fit to observations.

On the other hand Dravins, Lindgren, and Nordlund (1981) and Dravins (1988) have constructed a more sophisticated model of convection zone by actually solving the set of hydrodynamic equations for solar granulation. Because of constraints imposed by computer time such models have to be restricted in extent and hence, all scales of convection can not be included. However, such models give detailed convective velocity field and hence can be used to construct synthetic line profiles using the equation of radiative transfer. These 417 
models have essentially no free parameter and can provide a good test for the hypothesis of convective origin of spectral line asymmetries.

Antia and Pandey(1989, hereinafter paper I) considered a model of stellar convection zone in terms of linear convective modes. This model has the advantage of being simpler than the full simulation models and can provide velocity field over the entire stellar volume. Such stellar convection models can give useful insight about the scale of convection in various stars which could be used to construct the three dimensional simulation models.

The linear convective modes in the solar convection zone have been studied by Antia, Chitre, and Narasimha (1983) and it was found that there are two peaks in the growth rate versus horizontal wavelength plot which are in a reasonable agreement with the observed length and time scales of granulation and supergranulation. The linear stability theory used in this approach does not give the amplitudes of these modes which can only be determined by nonlinear processes. However, it is reasonable to assume that the convective flux will be transported by a combination of these modes. Narasimha and Antia (1982) have demonstrated that it is possible to construct a linear superposition of statistically independent unstable convective modes which reproduces convective flux as required by the mixing length theory over the entire convection zone. The resultant vertical velocities were found to be in a reasonable agreement with the observed granular velocities in the solar atmosphere. This may not provide a fully realistic model of stellar convection zone since it is based on the mixing length theory which itself is uncertain to a large extent. However, it can provide a reasonable first approximation to stellar convection zone.

Since it is not possible to estimate the relative phase of each of these convective modes which in any case cannot be constant. Hence, for simplicity we approximate the velocity and temperature fluctuation on the stellar surface as being due to a single convective mode. For this purpose we select the mode with largest amplitude of velocity and intensity fluctuations in the photospheric line formation region. Using the velocity field provided by the convection zone model we construct synthetic line profiles by integrating over the entire stellar surface. At each point on the stellar surface the line profile is assumed to be symmetric, the actual form being given by the usual Voigt function.

Following this approach Antia and Pandey(1989) constructed stellar envelope models for four stars i.e., the Sun, $\alpha$-Cen A, Arcturus and Procyon. The resulting line bisectors were found to be in a reasonable agreement with observed shapes but the line shifts were suppressed. In this paper the study has been extended to $\alpha$-Cen B and moreover the line shifts are also included.

\section{Numerical Results}

Following paper I, a convection zone model for $\alpha$-Cen B is constructed using a mixing length of $z+409 \mathrm{~km}$. The resulting model has a convection zone depth of $\approx 2 \times 10^{5} \mathrm{~km}$. The linear stability of this model is studied to find the convective modes. The turbulent prandtl number is assumed to be 0.33 in these calculations. The growth rate of these mode as a function of the degree of spherical harmonic $\ell$ is displayed in Fig. 1 . It can be seen that there are two peaks in the curve, the dominant peak around $\ell=400$ corresponds to a horizontal scale of $\approx 10^{4} \mathrm{~km}$ while the secondary peak around $\ell=90$ corresponds to a length scale of $\approx 4.5 \times 10^{4} \mathrm{~km}$. The corresponding time scales are $\approx 16$ and $130 \mathrm{hrs}$ respectively. Hence for $\alpha$-Cen B the granules should have a typical length scale of $10^{4} \mathrm{~km}$ and life-time of several hours. 
TABLE 1: Amplitude of convective modes at optical depth $=0.4$

\begin{tabular}{rrrc}
\hline$\ell$ & $\begin{array}{c}V_{r} \\
\left(\mathrm{~ms}^{-1}\right)\end{array}$ & $\begin{array}{c}V_{h} \\
\left(\mathrm{~ms}^{-1}\right)\end{array}$ & $F_{1} / F$ \\
\hline 1 & .01 & -20 & $2 \times 10^{-6}$ \\
2 & .04 & -69 & $8 \times 10^{-6}$ \\
4 & .09 & -97 & $2 \times 10^{-5}$ \\
8 & .34 & -166 & $2 \times 10^{-5}$ \\
16 & .73 & -223 & $2 \times 10^{-4}$ \\
24 & .94 & -216 & $3 \times 10^{-4}$ \\
36 & 1.76 & -270 & $5 \times 10^{-4}$ \\
54 & .32 & -37 & $1 \times 10^{-4}$ \\
80 & 2.46 & -283 & $1 \times 10^{-3}$ \\
120 & 19 & -829 & $4 \times 10^{-3}$ \\
180 & 74 & -118 & $2 \times 10^{-3}$ \\
270 & 1600 & -2510 & $4 \times 10^{-2}$ \\
400 & 1800 & -4600 & $8 \times 10^{-2}$ \\
450 & 61 & -217 & $4 \times 10^{-3}$ \\
\hline
\end{tabular}

To determine the amplitudes of these modes we obtain a linear superposition which fits the convective flux assumed in the mixing length model. The results are shown in Table 1 which gives the amplitude of velocity and flux perturbation at the level of optical depth 0.4 . It can be seen that the perturbations are dominated by $\ell=400$ convective mode. Unlike the case of the Sun or $\alpha$-Cen A there is no pronounced secondary peak in these amplitudes. Fig. 2 displays the convective flux profile obtained by superposing 14 modes listed in Table 1 . This figure also shows the convective flux profiles of some of the individual modes. It can be seen that the modes with different values of $\ell$ have peaks at different depths. Further, as explained in paper I we can identify the mixing length at a given depth with the width of flux profile which has peak at that depth. Fig. 3 displays the mixing length as a function of $\log P$, which can be compared with the width of convective flux profiles of individual modes marked by circles in the figure.

Using the velocity and flux perturbations due to the dominant convective mode the synthetic line profiles and bisectors are constructed as explained in paper I. The result is shown in Fig. 4 where the bisector is shown on a scale which is ten times the scale for line profile. For clarity Fig. 5 displays the line bisectors on an expanded scale where the curves have been shifted horizontally so that they do not overlap with each other. These line bisectors can be compared with the observed bisectors in Dravins(1987). It can be seen that the synthetic bisectors shape is in general agreement with the observed shapes.

While for other stars the line shift is not yet measured, but for the Sun the line shift is known to be of order of $400 \mathrm{~m} \mathrm{~s}^{-1}$. For the five typical line bisectors shown for the Sun from left to right in Fig. 6(a) of paper I the calculated line shifts are found to be respectively $439,313,358,298,202 \mathrm{~ms}^{-1}$. While the magnitude of these shifts is comparable to the observed values unfortunately the sign is not correct and all the lines shown in paper I are red shifted rather than the observed blue shifted lines. It is found that if the sign of $F_{1} / F$ used to construct line profiles is changed then the correct sign and value of line shift is obtained. However, in this case the line bisector essentially gets reflected and hence the shape does not agree with observations. It is not clear to me whether this discrepancy is due to some trivial point that I have missed in the calculations or is due to the inadequacy of the model.

For $\alpha$-Cen $A$ the red shifts for the typical profiles given in Fig. $6 \mathrm{~b}$ of paper I are 834, $789,629,612,1050 \mathrm{~m} \mathrm{~s}^{-1}$. For Arcturus the values are 169, -868, 170, -257, $-1066 \mathrm{~m}$ $\mathrm{s}^{-1}$. Thus for Arcturus it is possible to get both red shifted and blue shifted lines. This is probably due to the fact that modes with small values of $\ell$ dominate the fluctuations and for these modes the values may even depend on the orientation of axis assumed in the study. For Procyon all the typical profiles are blue shifted, with blue shifts of 672,810 , 1282,623 and $1070 \mathrm{~m} \mathrm{~s}^{-1}$ respectively. For $\alpha$-Cen B the typical profiles shown in Fig. 5 are red shifted by $288,314,291,433,319 \mathrm{~m} \mathrm{~s}^{-1}$ respectively from left to right. 


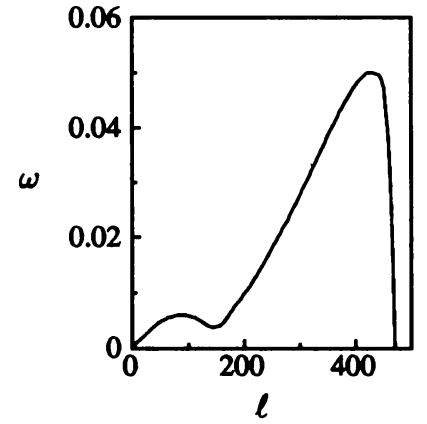

Fig. 1: The growth rate $\omega$ (in units of $\sqrt{\frac{3 G M}{4 \pi R^{3}}}$ ) of convective modes as a function of the degree of spherical harmonics $\ell$ for $\alpha$-Cen $B$.

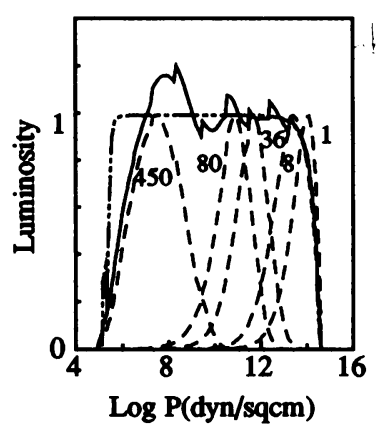

Fig. 2: The dashed curves show the convective luminosity due to individual convective modes for various values of $\ell$ as a function of $\log P$. The continuous curve shows superposed convective luminosity profile, while dot-dashed curve represents the model convective luminosity.

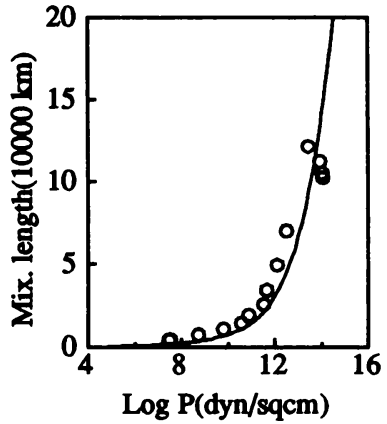

Fig. 3: The mixing length vs. logarithm of pressure for $\alpha$-Cen B. The width of the luminosity profile of various convective modes is indicated by circles.

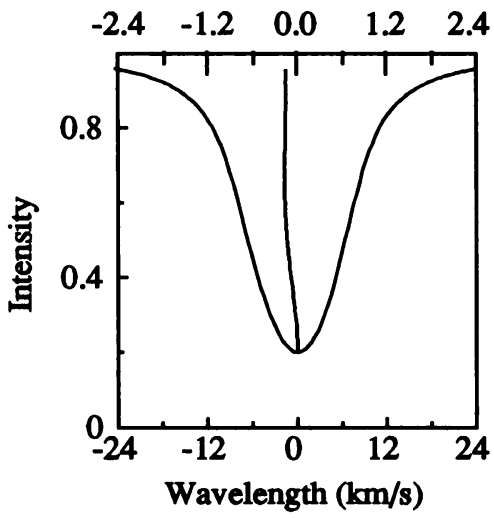

Fig. 4: Representative synthetic line profile and bisector for $\ell=400, V_{r}=2.5 \mathrm{~km}$ $\mathrm{s}^{-1}, V_{h}=-7 \mathrm{~km} \mathrm{~s}^{-1}$, and $F_{1} / F=-0.1$. The horizontal wavelength scale (in velocity units) refers to the line profile, while the ten times expanded upper scale refers to the bisector.

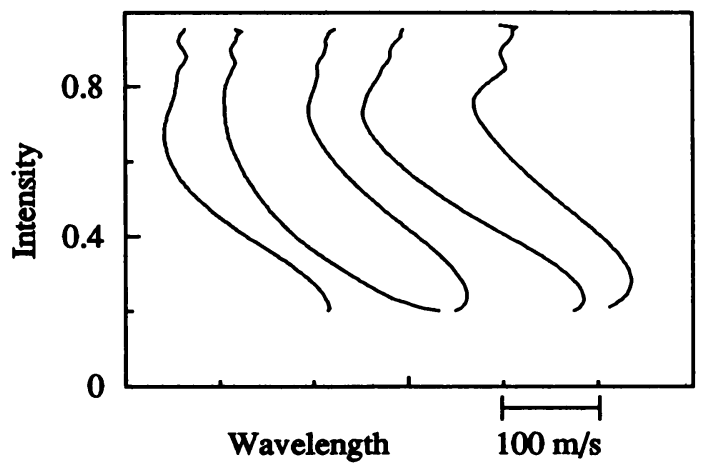

Fig. 5: Synthetic line bisectors for some typical values of the amplitudes of convective modes. The curves have been shifted horizontally to avoid overlapping. The values of $\left(\ell, V_{r}, V_{h}, F_{1} / F\right)$ for various curves from left to right are: $(400,2.5,-7,-0.1),(400,2,-8$, $-0.1),(400,1.5,-7,-0.2),(400,2,-7,-0.2),(400$, $2,-6,-0.2)$. Here $V_{r}$ and $V_{h}$ are in $\mathrm{km} \mathrm{s}^{-1}$.

\section{REFERENCES}

Antia, H. M., Chitre, S. M., and Narasimha, D. 1983, Mon. Not. R. Astr. Soc., 204, 865. Antia, H. M., and Pandey, S. K. 1989, Astrophys. J., 252 (in press).

Dravins, D. 1987, Astr. Ap., 172, 211.

Dravins, D. 1988, in R. J. Rutten, G. Severino, eds. Solar and Stellar Granulation.

Dravins, D., Lindgren, L., and Nordlund, А. 1981, Astr. Ap., 96, 345.

Gray, D. F., and Toner, C. G. 1985, Publ. Astron. Soc. Pacific, 97, 543.

Narasimha, D., and Antia, H. M. 1982, Ap. J., 262, 358. 